\title{
Toward a Membrane-Centric Biology
}

\author{
Yan Shi ${ }^{1,2,3,4,5 *}$ and Hefei Ruan ${ }^{1,2,3,4}$ \\ ${ }_{1}^{1}$ Tsinghua-Peking University Joint Center for Life Sciences, Tsinghua University, Beijing, China, ${ }^{2}$ Department of Basic Medical \\ Sciences, Tsinghua University, Beijing, China, ${ }^{3}$ Institute for Immunology, Tsinghua University, Beijing, China, ${ }^{4}$ Beijing Key Lab \\ for Immunological Research on Chronic Diseases, School of Medicine, Tsinghua University, Beijing, China, ${ }^{5}$ Department of \\ Microbiology, Immunology and Infectious Diseases, Snyder Institute, University of Calgary, Calgary, AB, Canada
}

OPEN ACCESS

Edited by:

Junji Xing,

Houston Methodist Research Institute,

United States

Reviewed by:

Chaofeng Han,

State Key Laboratory of Medical Immunology, Second Military Medical

University, China

Chunfu Zheng,

Fujian Medical University, China

*Correspondence:

Yan Shi

yanshiemail@mail.tsinghua.edu.cn

Specialty section:

This article was submitted to

Molecular Innate Immunity,

a section of the journal

Frontiers in Immunology

Received: 21 May 2020

Accepted: 16 July 2020

Published: 09 September 2020

Citation:

Shi Y and Ruan H (2020) Toward a

Membrane-Centric Biology.

Front. Immunol. 11:1909.

doi: 10.3389/fimmu.2020.01909
With advancements of modern biophysical tools and superresolution imaging, cell biology is entering a new phase of research with technological power fitting for membrane dynamics analyses. However, our current knowledge base of cellular signaling events is mostly built on a network of protein interactions, which is incompatible with the essential roles of membrane activities in those events. The lack of a theoretical platform is rendering biophysical analyses of membrane biology supplementary to the protein-centric paradigm. We hypothesize a framework of signaling events mediated by lipid dynamics and argue that this is the evolutionarily obligatory developmental path of cellular complexity buildup. In this framework, receptors are the late comers, integrating into the pre-existing membrane based signaling events using their lipid interface as the point of entry. We further suggest that the reason for cell surface receptors to remain silent at the resting state is via the suppression effects of their surrounding lipids. The avoidance of such a suppression, via ligand binding or lipid domain disruption, enables the receptors to autonomously integrate themselves into the preexisting networks of signaling cascades.

Keywords: plasma membrane, lipid rafts, evolution, receptor ligand model, lipid interaction, receptor activation mechanism

\section{INTRODUCTION}

The main goal of this piece is to gather sufficient consensus regarding how biophysicists, or other specialists so inclined, may approach life science research with a stronger footing of legitimacy. In recent years, with advancements in superresolution imaging and computational biology, biophysicists are given enormous probing power in our life science work. In comparison, traditional biologists using more conventional tools are still making ground-breaking discoveries at a pace appreciably faster than most of us. A sobering dichotomy is evident that findings made with biophysical approaches are being regarded as "supplemental" to other paradigms. Using T cell biology as an example, whereas many papers have been published in this area regarding membrane behavior upon $\mathrm{T}$ cell receptor activation, the whole theoretical framework of $\mathrm{T}$ cell activation can be completely explained without any reference to biophysical properties. Points of interest in biophysics or membrane biology are generated to explain the details of how polypeptides work. While we know for certain that this cannot be true, our strongest protest may be the insistence that "no signaling can be fully understood without its membrane platform." C'est la vie, such a defensive stand will not change the "outsider looking-in" mentality. We need to move biophysics and membrane biology to the frontlines of biological research. The questions are "why hasn't it happened?" and "what is the main roadblock?" 


\section{MAIN TEXT}

The current state of biological research is protein-centric. The cause lies in its history and the availability of investigative tools. Yet, conceptual inertia is not beyond reproach. Our inability to delineate biological events with models built from membrane biology is the core deficiency. It is time that we collectively reflect on this dilemma. In this opinion piece, we make a call for change.

\section{Membrane Structure, Critical Behavior, and Their Preservation in Biology}

Let's start with the eukaryotic membrane. In the 70s, Singer and Nicolson presented the mosaic model in which the membrane bilayer was regarded as a fluid mixture of lipids and proteins (1). With many years of work into the heterogeneity of vesicular and plasma membranes, Kai Simons et al. in 1997 proposed the concept of ordered and disordered membrane phases generically known as the lipid raft theory (2). Aki Kusumi, mainly using particle tracking, refined this model with an additional detail that lipid domains are stabilized by membrane lipid binding to cortical cytoskeleton, or the picket and fence model (3). As those theories are discussed at length elsewhere and readers of this writing are well versed in this stream of concepts, we simplify our discussion with the most accepted membrane model (4). Eukaryotic membrane inner leaflets are occupied by mostly phospholipids with negative charge and are active in signal exchange in abundance. In comparison the outer leaflets are structurally dynamic. There, sphingolipids and gangliosides are more enriched, perhaps due to their enlarged head groups more suited to the positive curvature. Cholesterol, nimble in size and low in charge, is free to move laterally (5) or change leaflets via overcoming the energy barrier set by hydrophobic core of the bilayer (6). The rendezvous of sphingolipids, cholesterol, and saturated phospholipids at physiological temperatures forms the structure of ordered lipid domains. The formation of ordered vs. disordered lipid domains can be explained by the combined entropic diffusion and energy conservation in special lipid pairing $(7,8)$. Remarkably, this feature is common to all eukaryotes despite the vast different collections of lipid species in distinct cell types. About $5 \%$ of genes are dedicated to maintain it (4). As current efforts have not been able to fully mimic domains found in live cells with defined lipids, the remarkable preservation implies an extreme cellular dedication in their maintenance. This point alone should give us a strong clue that this is something central to all aspects of eukaryotic biology.

Another intrigue of the lipid domains is the critical behavior which refers to the state where at physiological temperature, lipid domain formation (demixing) and dissolution (mixing) are at a critical point (7). This feature, coupled to cytoskeletal association, was vividly demonstrated with STED superresolution microscopy, and with a clear linear correlation to the temperature (9). Such a delicate feature allows large phase transition with minimal energy input. For instance, minute disturbance of receptor ligand interaction may force such a phase change, an ingenious system of signal amplification (receptor ligation can be viewed as a localized suppression of entropy, or cooling). We shall return to this point later. Nevertheless, it should be noted that such a behavior is unimaginable in a cohort of protein molecules.

\section{Current Status of Understanding}

Since the proposal of lipid rafts, biologists have tried to incorporate this feature into their models of membrane signaling. To circumvent the optical diffraction limit which makes visual observations of resting cell lipid rafts impossible, two surrogates have been developed. One is to isolate detergent-resistant membrane domains, hoping to capture proteins associated with or free from lipid rafts at the moment of cell lysis (10). The other, used by some, is to observe domain coalescence at the point of "signalosome" formation (11) or visible lipid domains found on GPMV (12). One of the most influential conclusions is the partition of protein molecules into different phases of membrane domains. From those experiments, it was understood that the transition of those protein molecules with reference to lipid domains is associated with their state of activation. Some are activated in disordered phase, such as EGFR (13), while in others transition into or residence inside the ordered domain is required for their activation, such as death receptor Fas, IFN $\gamma \mathrm{R}$, and Wnt receptor (14-16). In addition, protein signaling complex formation with the participation of numerous components is also controlled by the coalescence of lipid rafts, such as in TCR activation (17). Regardless of the study subject, in a proteincentric world, those events are regarded as the consequence of receptor ligation and protein-protein interaction, which is taken as the driving force of lipid domain alteration.

Those observations, however, are not without their own peril. First, the selection of detergents has a tremendous effect on the observed association, demanding caution in data interpretation. Perhaps more importantly, this "snap photo" approach will leave out spatial temporal regulation. Using TCR as an example, the signaling is mediated by TCR ligation by the MHC/peptide complex, yet the signal is initiated at Src family kinase activation of the tyrosine residues in the ITAM motifs. Thereafter, the signal has several bifurcations or multiplications; some of downstream events such as Lck and LAT are clearly dependent on lipid domains (18), while others such as TCR itself and CD45 are not $(19,20)$. Likely due to those technical limitations, some conclusions are not always in agreement. In a remarkable demonstration of collegiality among biophysicists, those differences are accepted as limits of one's own research unable to explain seemingly contradicting results. In fact, those differences en masse reflect the lack of more sophisticated tools as well as a biophysical explanation of how membranes work in this setting. Happily, the constraint posed by tool selection is being rapidly lifted in recent years. A particular case in point is the newly gained ability to study the dynamics of lipid rafts on the cell membrane, which in our opinion is the technological foundation to introduce membrane dynamics into core concepts of biology.

\section{Evidence of Membrane Lipid Interface Is a Biological Switch}

Imagine a simplest eukaryotic cell with no cell surface receptor and driven by a few signaling pathways that support the basic 
biology. All those regulations are anchored to membrane sensing. Earliest multicellular animals were found about 600 million years ago. Shortly after, about 550 million years ago, life rapidly diversified during the Cambrian explosion. Around that time two rounds of whole genome duplication likely provided a genetic playground for the emergence of vertebrates (20). It is hard to imagine that receptor ligand interactions would have been the dominant way of communication prior to these junctures. The definition of receptor-ligand interaction is that they must be evolutionarily coupled. As a single cell is exposed to an unmalleable environment, this co-evolution lacked a driving force. On the flip side, receptor-independent sensing of environment, such as phagocytosis $(21,22)$, was a daily occurrence that had propelled the evolution for at least 1.4 billion years. Many prominent signaling pathways came before this time, including GTPase (23), MAPK (24), phagocytosis (21), TNFRF (25), Jak/Stat (26) pathways, metalloproteases, (27) and metabolic events with a possible exception of Wnt pathway (28). All those pathways are regulated by membrane events. In our own research, we first discovered that solid particle binding to plasma membrane induces the accumulation of lipid rafts which triggers phagocytosis $(29,30)$. Based on this finding, we further revealed that immune receptors had evolved out of a primordial phagocytic signaling that uses the membrane anchoring protein moesin to sense the PIP2 accumulation in the inner leaflet as a result of particle binding. Moesin binding to PIP2 opens its ITAM motif, for downstream signaling, including Syk and PI3K. Remarkably, all those events are used verbatim in immune signaling of all classes, including BCR, TCR, and Fc receptors (21). Therefore, the adaptive immunity hijacked the machinery of the ancient phagocytosis following membrane sensing. If the intracellular events are regulated by membrane activities, what argues against the notion that modern immune receptors initiate signaling with the same mechanism?

Let's look at another set of events observed by most if not all whom have attempted: the consequence of lipid domain perturbation, mostly in the form of cholesterol depletion. EGFR (31-33), TNFR1 (34), TLRs $(35,36)$, TCR $(19,37,38)$, TGFßR (39-41), and shedding events (42-44) are triggered by lipid domain disruption. Figure 1 illustrates our own findings. There are certainly good examples where clustering toward lipid rafts is induced by ligand binding. Linked to the observation that those receptors are sequestered in their own lipid environment at the resting state, one can reasonably predict: 1 . Some receptors are self-activating depending on the lipid environment or phase transition. 2. For one receptor to be accepted as useful contributor to biology, it would have to obey the suppression of the membrane. Therefore, membrane lipid phases are an ingrained tool of suppression of receptors. Kai Simons noted that due to the size of lipid domains, each raft would contain very few polypeptides (45). Therefore, those receptors are blocked by their spatial separation. Once the blockage is released, such as in the case of domain disruption, they become activated. Fessler and Parks used a number of examples to show how lipid perturbation itself is sufficient to activate many receptors (46). Yet, they stopped at the last step to connect the final dots that lipid perturbation-mediated receptor activation and ligand/receptor-based activation may be fundamentally the same at least for some receptor families. To extrapolate this idea further, we can imagine that a receptor ligation interaction, rather than bringing in certain conformations to accommodate their interaction binding partners, could easily regulate its lipid interface to avoid the suppression. In light of the critical behavior in membrane lipids, such a minute change can cause the clustering of signaling molecules as a consequence of lipid domain alteration at the energy level fitting for receptor ligand interaction. This hypothesis, with modern tools, should be testable in situ or on a model membrane without the need of biological feedback, which often causes protein-centric analyses becoming embroiled in incessant cycles of amplification. If a receptor/lipid interface event is established as being autonomous, this binary regulation certainly carries an enormous power of prediction, with a simplistic elegance not seen in sometimes chaotic search of how each protein receptor works with its downstream partners (Figure 2). At minimum, it explains why several thousand signaling receptors can stay silently together on a cell, and during an activation event, several signaling cascades are triggered at the same time, as those involved are likely gated by their own membrane sequestration. This is not to say that receptors are mere puppets in this chain of events; they certainly play their role in the clustering and their own complex formation as they also possess ranges of lipid specificities and membrane dynamics $(47,48)$. But the activation initiation point can be explained by lipid receptor interface, or the sum of "collectives" of protein-lipid interaction following the introduction of a ligand.

\section{Toward a Simple Beginning}

Our lab has preliminary data to suggest that "suppression avoidance" is a core mechanism of some cell death receptors, and the cellular signaling is initiated at the simple phase change between the receptor and its surrounding lipid species. This type of effort, while fulfilling the common wisdom that receptor activation is responding to its ligand, is probably also suited to explain some activation triggers, particularly those that are not protein in nature. In such a scenario, many "ligands" can activate their "receptors" via lipid alteration without the need of direct engagement. For instance, a long acyl chain fatty acid can alter the domain features, which allows a particularly strong signaling receptor to become activated in response to lipid domain change. Macroscopically, this would look like a perfect receptor/ligand interaction. Current dogma would require the search of how this pair of receptor/ligand works, but the "suppression avoidance" model would relieve ourselves from this futility. In fact, some of the low-hanging fruits should be easy to spot.

We are not arguing against the vast network of protein signaling in biology. However, from an evolutionary perspective, membrane triggered events should be highly relevant and they set the basic signaling principles in the cell. The numerical imbalance between the vast number of cell/vesicle surface receptors and limited signaling pathways clearly tells us that the former hijacked the latter, to develop the mesmerizingly 


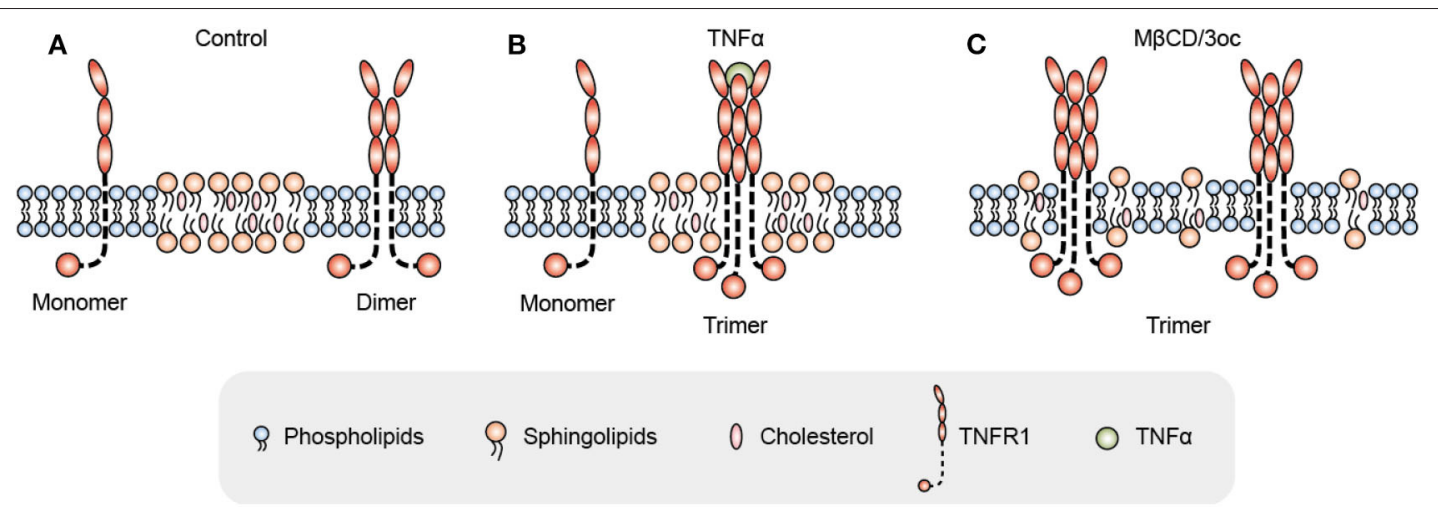

FIGURE 1 | Proposed mechanism for TNFR1 signal activation initiated as a negotiation between the receptor and its surrounding lipid species. (A) In the resting state, TNFR1 mainly exists as monomers and dimers on the cell membrane under the suppression of dense lipid rafts. (B) Under TNF $\alpha$ ligand stimulation, TNFR1 negotiates with lipid domains by changing its transmembrane domain conformation, and then enters lipid rafts for trimerization. (C) When the lipid rafts are disrupted by $\mathrm{M} \beta \mathrm{CD}$ or 3oc [N-(3-oxododecanoyl) homoserine lactone], TNFR1 spontaneously trimerizes and activates signals without ligands as the membrane suppression is relieved.

Single cell life form

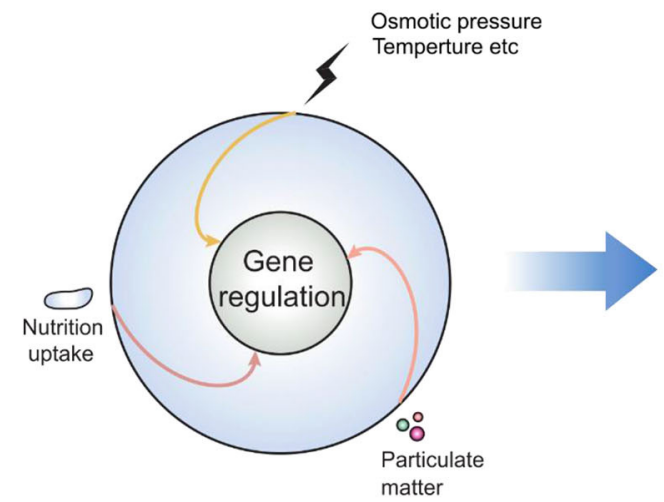

Complex muticellular organism

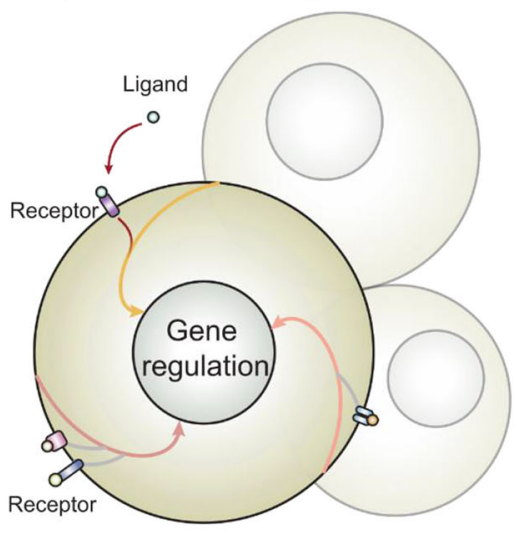

FIGURE 2 | How receptors may enter the preexisting signal cascades: as many signaling events present in modern day eukaryotic cells predate the multicellular life forms, those enclosed single cells interacted with the environment via lipid membrane. Those signal cascades are therefore coupled to membrane sensing, particularly in response to lipid domain alteration. New receptors cannot reinvent a new signal cascade; rather than producing protein molecules as adaptors, they can also regulate their own lipid interface, which is the legitimate and built-in mechanism of cell activation.

complex activation patterns in modern eukaryotic cells. Similar to the preservation of amino acid codons, those basic signaling events cannot be altered in the biological continuum. Then it is reasonable to question how the late comers, the receptor-ligand interaction, came into the theme. For our purpose, if they also use the lipid interface as the initiation point, then we have the theoretical prowess to establish a model toward a membranebased biology, to smooth out rough edges and peculiarities in the protein-centric paradigm.

This opinion piece may be deemed inaccurate or even false in the future. However, as a research discipline with cutting edge tools and deals with some of the most autonomous events that formed the platform for other late developed biology, our collective attempt to create a landscape for this new frontier, no matter how juvenile at the beginning, is certainly worthwhile.

\section{DATA AVAILABILITY STATEMENT}

The original contributions presented in the study are included in the article/supplementary material, further inquiries can be directed to the corresponding author/s.

\section{AUTHOR CONTRIBUTIONS}

YS conceptualized the theory and wrote the manuscript with assistance from HR. All authors contributed to the article and approved the submitted version.

\section{FUNDING}

YS was supported by the joint Peking-Tsinghua Center for Life Sciences, the National Natural Science Foundation of China 
General Program (31370878), State Key Program (31630023), and Innovative Research Group Program (81621002), by grants from CIHR (PJT-156334 and PJT-166155) and NSERC (RGPIN/03748-2018). HR was supported by China Postdoctoral Science Foundation (No. 2019M650718).

\section{REFERENCES}

1. Singer SJ, Nicolson GL. The fluid mosaic model of the structure of cell membranes. Science. (1972) 175:720-31. doi: 10.1126/science.175.4023.720

2. Simons K, Ikonen E. Functional rafts in cell membranes. Nature. (1997) 387:569-72. doi: 10.1038/42408

3. Ritchie $\mathrm{K}$, Iino $\mathrm{R}$, Fujiwara $\mathrm{T}$, Murase $\mathrm{K}$, Kusumi A. The fence and picket structure of the plasma membrane of live cells as revealed by single molecule techniques (Review). Mol Membr Biol. (2003) 20:13-8. doi: 10.1080/0968768021000055698

4. van Meer G, Voelker DR, Feigenson GW. Membrane lipids: where they are and how they behave. Nat Rev Mol Cell Biol. (2008) 9:112-24. doi: $10.1038 / \mathrm{nrm} 2330$

5. Scheidt HA, Huster D, Gawrisch K. Diffusion of cholesterol and its precursors in lipid membranes studied by $1 \mathrm{H}$ pulsed field gradient magic angle spinning NMR. Biophys J. (2005) 89:2504-12. doi: 10.1529/biophysj.105.062018

6. Gu RX, Baoukina S, Tieleman DP. Cholesterol flip-flop in heterogeneous membranes. J Chem Theory Comput. (2019) 15:2064-70. doi: $10.1021 /$ acs.jctc.8b00933

7. Machta BB, Papanikolaou S, Sethna JP, Veatch SL. Minimal model of plasma membrane heterogeneity requires coupling cortical actin to criticality. Biophys J. (2011) 100:1668-77. doi: 10.1016/j.bpj.2011.02.029

8. Crow KD, Wagner GP, SMBE Tri-National Young Investigators. Proceedings of the SMBE Tri-National Young Investigators' Workshop 2005. What is the role of genome duplication in the evolution of complexity and diversity? $\mathrm{Mol}$ Biol Evol. (2006) 23:887-92. doi: 10.1093/molbev/msj083

9. Honigmann A, Sadeghi S, Keller J, Hell SW, Eggeling C, Vink R. A lipid bound actin meshwork organizes liquid phase separation in model membranes. Elife. (2014) 3:e01671. doi: 10.7554/eLife.01671.013

10. Thomas S, Preda-Pais A, Casares S, Brumeanu TD. Analysis of lipid rafts in T cells. Mol Immunol. (2004) 41:399-409. doi: 10.1016/j.molimm.2004.03.022

11. Dykstra M, Cherukuri A, Sohn HW, Tzeng SJ, Pierce SK. Location is everything: lipid rafts and immune cell signaling. Annu Rev Immunol. (2003) 21:457-81. doi: 10.1146/annurev.immunol.21.120601.141021

12. Baumgart T, Hammond AT, Sengupta P, Hess ST, Holowka DA, Baird BA, et al. Large-scale fluid/fluid phase separation of proteins and lipids in giant plasma membrane vesicles. Proc Natl Acad Sci USA. (2007) 104:3165-70. doi: $10.1073 /$ pnas.0611357104

13. Irwin ME, Mueller KL, Bohin N, Ge Y, Boerner JL. Lipid raft localization of EGFR alters the response of cancer cells to the EGFR tyrosine kinase inhibitor gefitinib. J Cell Physiol. (2011) 226:2316-28. doi: 10.1002/jcp.22570

14. Gajate C, Mollinedo F. Lipid raft-mediated Fas/CD95 apoptotic signaling in leukemic cells and normal leukocytes and therapeutic implications. J Leukoc Biol. (2015) 98:739-59. doi: 10.1189/jlb.2MR0215-055R

15. Blouin CM, Hamon Y, Gonnord P, Boularan C, Kagan J, De Lesegno C V, et al. Glycosylation-Dependent IFN- $\gamma \mathrm{R}$ partitioning in lipid and actin nanodomains is critical for JAK activation. Cell. (2016) 166:920-34. doi: 10.1016/j.cell.2016.07.003

16. Sezgin E, Azbazdar Y, Ng XW, Teh C, Simons K, Weidinger G, et al. Binding of canonical Wnt ligands to their receptor complexes occurs in ordered plasma membrane environments. FEBS J. (2017) 284:2513-26. doi: $10.1111 /$ febs. 14139

17. Jury EC, Flores-Borja F, Kabouridis PS. Lipid rafts in $T$ cell signalling and disease. Semin Cell Dev Biol. (2007) 18:608-15. doi: 10.1016/j.semcdb.2007.08.002

18. Razzaq TM, Ozegbe P, Jury EC, Sembi P, Blackwell NM, Kabouridis PS. Regulation of T-cell receptor signalling by membrane microdomains. Immunology. (2004) 113:413-26. doi: 10.1111/j.1365-2567.2004.01998.x

\section{ACKNOWLEDGMENTS}

We thank Drs. Erdinc Sezgin, Jack Wei Chen, Xiaoyu Hu, and Yonghui Zhang for discussion and feedback. We thank Ying Xu for the drawings.

19. Swamy M, Beck-Garcia K, Beck-Garcia E, Hartl FA, Morath A, Yousefi O S, et al. A cholesterol-based allostery model of $\mathrm{T}$ cell receptor phosphorylation. Immunity. (2016) 44:1091-101. doi: 10.1016/j.immuni.2016.04.011

20. Zhang M, Moran M, Round J, Low TA, Patel VP, Tomassian T, et al. CD45 signals outside of lipid rafts to promote ERK activation, synaptic raft clustering, and IL-2 production. J Immunol. (2005) 174:1479-90. doi: 10.4049/jimmunol.174.3.1479

21. Mu L, Tu Z, Miao L, Ruan H, Kang N, Hei Y, et al. A phosphatidylinositol 4,5bisphosphate redistribution-based sensing mechanism initiates a phagocytosis programing. Nat Commun. (2018) 9:4259. doi: 10.1038/s41467-01806744-7

22. Yutin N, Wolf MY, Wolf YI, Koonin EV. The origins of phagocytosis and eukaryogenesis. Biol Direct. (2009) 4:9. doi: 10.1186/1745-6150-4-9

23. Harris SD. Cdc42/Rho GTPases in fungi: variations on a common theme. Mol Microbiol. (2011) 79:1123-7. doi: 10.1111/j.1365-2958.2010.07525.x

24. Li M, Liu J, Zhang C. Evolutionary history of the vertebrate mitogen activated protein kinases family. PLoS ONE. (2011) 6:e26999. doi: 10.1371/journal.pone.0026999

25. Quistad SD, Traylor-Knowles N. Precambrian origins of the TNFR superfamily. Cell Death Discov. (2016) 2:16058. doi: $10.1038 /$ cddiscovery.2016.58

26. Liongue C, Ward AC. Evolution of the JAK-STAT pathway. JAKSTAT. (2013) 2:e22756. doi: 10.4161/jkst.22756

27. Fanjul-Fernández M, Folgueras AR, Cabrera S, López-Otín C. Matrix metalloproteinases: evolution, gene regulation and functional analysis in mouse models. Biochim Biophys Acta. (2010) 1803:3-19. doi: 10.1016/j.bbamcr.2009.07.004

28. Holstein TW. The evolution of the Wnt pathway. Cold Spring Harb Perspect Biol. (2012) 4:a007922. doi: 10.1101/cshperspect.a007922

29. Ng G, Sharma K, Ward SM, Desrosiers MD, Stephens LA, Schoel WM, et al. Receptor-independent, direct membrane binding leads to cell-surface lipid sorting and Syk kinase activation in dendritic cells. Immunity. (2008) 29:807-18. doi: 10.1016/j.immuni.2008.09.013

30. Flach TL, Ng G, Hari A, Desrosiers MD, Zhang P, Ward SM, et al. Alum interaction with dendritic cell membrane lipids is essential for its adjuvanticity. Nat Med. (2011) 17:479-87. doi: 10.1038/ nm.2306

31. Chen X, Resh MD. Cholesterol depletion from the plasma membrane triggers ligand-independent activation of the epidermal growth factor receptor. J Biol Chem. (2002) 277:49631-37. doi: 10.1074/jbc.M208327200

32. Furuchi T, Anderson RG. Cholesterol depletion of caveolae causes hyperactivation of extracellular signal-related kinase (ERK). J Biol Chem. (1998) 273:21099-104. doi: 10.1074/jbc.273.33.21099

33. Lambert S, Vind-Kezunovic D, Karvinen S, Gniadecki R. Ligand-independent activation of the EGFR by lipid raft disruption. J Invest Dermatol. (2006) 126:954-62. doi: 10.1038/sj.jid.5700168

34. Song D, Meng J, Cheng J, Fan Z, Chen P, Ruan H, et al. Pseudomonas aeruginosa quorum-sensing metabolite induces host immune cell death through cell surface lipid domain dissolution. Nat Microbiol. (2019) 4:97-111. doi: 10.1038/s41564-018-0290-8

35. Smoak KA, Aloor JJ, Madenspacher J, Merrick BA, Collins JB, Zhu X, et al. Myeloid differentiation primary response protein 88 couples reverse cholesterol transport to inflammation. Cell Metab. (2010) 11:493-502. doi: 10.1016/j.cmet.2010.04.006

36. Jackson SK, Abate W, Parton J, Jones S, Harwood JL. Lysophospholipid metabolism facilitates Toll-like receptor 4 membrane translocation to regulate the inflammatory response. J Leukoc Biol. (2008) 84:86-92. doi: 10.1189/jlb.0907601 
37. Rouquette-Jazdanian AK, Pelassy C, Breittmayer JP, Aussel C. Full CD3/TCR activation through cholesterol-depleted lipid rafts. Cell Signal. (2007) 19:1404-18. doi: 10.1016/j.cellsig.2007.01.015

38. Mahammad S, Dinic J, Adler J, Parmryd I. Limited cholesterol depletion causes aggregation of plasma membrane lipid rafts inducing $\mathrm{T}$ cell activation. Biochim Biophys Acta. (2010) 1801:625-34. doi: 10.1016/j.bbalip.2010. 02.003

39. Shapira KE, Ehrlich M, Henis YI. Cholesterol depletion enhances TGF- $\beta$ Smad signaling by increasing c-Jun expression through a PKR-dependent mechanism. Mol Biol Cell. (2018) 29:2494-507. doi: 10.1091/mbc.E18-03-0175

40. Chen CL, Huang SS, Huang JS. Cholesterol modulates cellular TGF-beta responsiveness by altering TGF-beta binding to TGF-beta receptors. J Cell Physiol. (2008) 215:223-33. doi: 10.1002/jcp.21303

41. Chen CL, Liu IH, Fliesler SJ, Han X, Huang SS, Huang JS. Cholesterol suppresses cellular TGF-beta responsiveness: implications in atherogenesis. J Cell Sci. (2007) 120(Pt 20):3509-21. doi: 10.1242/jcs. 006916

42. Matthews V, Schuster B, Schütze S, Bussmeyer I, Ludwig A, Hundhausen C, et al. Cellular cholesterol depletion triggers shedding of the human interleukin-6 receptor by ADAM10 and ADAM17 (TACE). J Biol Chem. (2003) 278:3882939. doi: $10.1074 /$ jbc.M210584200

43. von Tresckow B, Kallen KJ, von Strandmann EP, Borchmann P, Lange H, Engert A, et al. Depletion of cellular cholesterol and lipid rafts increases shedding of CD30. J Immunol. (2004) 172:4324-31. doi: 10.4049/jimmunol.172.7.4324
44. Murai T, Maruyama Y, Mio K, Nishiyama H, Suga M, Sato C. Low cholesterol triggers membrane microdomain-dependent CD44 shedding and suppresses tumor cell migration. J Biol Chem. (2011) 286:1999-2007. doi: $10.1074 /$ jbc.M110.184010

45. Simons K, Toomre D. Lipid rafts and signal transduction. Nat Rev Mol Cell Biol. (2000) 1:31-9. doi: 10.1038/35036052

46. Fessler MB, Parks JS. Intracellular lipid flux and membrane microdomains as organizing principles in inflammatory cell signaling. J Immunol. (2011) 187:1529-35. doi: 10.4049/jimmunol.1100253

47. Contreras FX, Ernst AM, Haberkant P, Björkholm P, Lindahl E, Gönen B, et al. Molecular recognition of a single sphingolipid species by a protein's transmembrane domain. Nature. (2012) 481:525-9. doi: 10.1038/nature10742

48. Lozano MM, Hovis JS, Moss FR 3rd, Boxer SG. Dynamic reorganization and correlation among lipid raft components. J Am Chem Soc. (2016) 138:999610001. doi: 10.1021/jacs.6b05540

Conflict of Interest: The authors declare that the research was conducted in the absence of any commercial or financial relationships that could be construed as a potential conflict of interest.

Copyright $(02020$ Shi and Ruan. This is an open-access article distributed under the terms of the Creative Commons Attribution License (CC BY). The use, distribution or reproduction in other forums is permitted, provided the original author(s) and the copyright owner(s) are credited and that the original publication in this journal is cited, in accordance with accepted academic practice. No use, distribution or reproduction is permitted which does not comply with these terms. 